\title{
Characterization of the Effects of Silver Nanoparticles on Liver Cell Using HR-MAS NMR Spectroscopy
}

\author{
Siwon Kim, Sosun Kim, Sangmi Lee, Bobae Kwon, Jinhee Choi, ${ }^{\dagger}$ Jin Won Hyun, ${ }^{\ddagger}$ and Suhkmann Kim* \\ Department of Chemistry and Chemistry Institute for Functional Materials, Pusan National University, Busan 609-735, Korea \\ *E-mail: suhkmann@pusan.ac.kr \\ ${ }^{\dagger}$ Faculty of Environmental Engeering, University of Seoul, Seoul 130-743, Korea \\ \$School of Medicine and Applied Radiological Science Research Institute, Jeju National University, Jeju 690-756, Korea \\ Received April 12, 2011, Accepted April 25, 2011
}

\begin{abstract}
AgNPs (silver nanoparticles) has been widely used for the commercial products, which have antimicrobial agent, medical devices, food industry and cosmetics. Despite, AgNPs have been reported as toxic to the mammalian cell, lung, liver, brain and other organs and many researchers have investigated the toxicity of AgNPs. In this study, we investigated toxicity of the AgNPs to the liver cell using metabolomics based on HRMAS NMR (High Resolution Magic Angle Spinning Nuclear Magnetic Resonance) technics, which could apply to the intact tissues or cells, to avoid the sample destruction. Target profiling and multivariative statistical analysis were performed to analyze the $1 \mathrm{D}{ }^{1} \mathrm{H}$ spectrum. The results show that the concentrations of many metabolites were affected by the AgNPs in the liver cell. The concentrations of glutathione (GSH), lactate, taurine, and glycine were decreased and most of amino acids, choline analogues, and pyruvate were increased by the AgNPs. Moreover, the levels of the metabolites were recovered upto similar level of metabolites in the normal cell by the pre-treatment of NAC, external antioxidant. The results suggest that the depletion of the GSH by the AgNPs might induce the conversion of lactate and taurine to the pyruvate.
\end{abstract}

Key Words : Toxicity, Silver nanoparticles, HR-MAS NMR, Metabolomics

\section{Introduction}

Nanoparticles (NPs), which describes as particles with lengths that range from 100 to $2500 \mathrm{~nm}$ of fine particles and sized between 1 and $100 \mathrm{~nm}$ of ultrafine particles in two or three dimensions. In particular, NPs have unique properties related in size of particle, which is difference in bulk materials. $^{1-3}$ It has been used in various industries for bio application, such as drug delivery, molecular diagnostics and biosensors. ${ }^{4}$ Although NPs can induce some health problems for example lung injury, effect on human immune cells and cytotoxicity. 5 ,6 Among them, silver nano particles (AgNPs) are one of the most widely used commercial products, which have antimicrobial agent, medical devices, food industry and cosmetics. ${ }^{7-10}$ Despite, AgNPs have been reported as toxic to the mammalian cell, lung, liver, brain and other organs. ${ }^{11,12}$ Recent studies suggested that AgNP cytotoxicity is related with oxidative stress in human hepatoma cells (Hep G2). ${ }^{13}$

Oxidative stress (OS) is standing for the steady state level of oxidative damage in a cell, tissue, or organ caused by the reactive oxygen species (ROS) ${ }^{14,15}$ The damage is possible to affect the specific molecule or entire organism. In addition, ROS is related with cell death and trigger of apoptosis. ROS is included in free radicals and peroxide, such as hydrogen peroxide $\left(\mathrm{H}_{2} \mathrm{O}_{2}\right)$, the free radicals superoxide $\left(\mathrm{O}_{2}{ }^{-}\right)$and hydroxyl radical (HO-). In addition, ROS can damage structure and function of biomolecules such as DNA, protein and lipids. The amount of the ROS in the cell could be detected by the level of GSH which is depleted while oxidized to glutathione disulfide (GSSG) by the ROS. ${ }^{16}$

Newly, '-omic' technology, called metabolomics, has useful method to identify the small molecules and pathways, which is applied to biological samples, such as blood, urine, biofluid, tissues and cells. ${ }^{17-20}$ Currently, metabolomics are utilized by commonly two methods, mass spectrometry (MS) or nuclear magnetic resonance (NMR) spectroscopy. In particular, introduction of high resolution magic angle spinning (HR-MAS) NMR spectroscopy has made it possible to analyze the metabolite compounds in the intact tissues or cells to avoid sample destruction. The magic angle, $\theta=$ $54.7^{\circ}$, is induced by the line broadening effects of chemical shift anisotropy and dipolar coupling could reduce. The result of fast spinning obtains notable improvement of the resolution of spectrum. Consequently, this technique acquires high resolution spectra. Previous studies show the successful results in biological tissue, such as, human liver, rat liver, mouse intestine, human breast and human brain. ${ }^{21-23}$

NMR-based metabolomics has two routes for analysis methods, which are target profiling and pattern recognition of spectrum. Target profiling provides new approach to explore the specific metabolic effects of several conditions in biological systems. Second method is different approach to interpreting metabolic profiles of spectra. In this case, only recorded spectra patterns and intensities are compared to distinguish the differences of their metabolite profiles.

In our previous studies, AgNP induced oxidative cell damage in human liver cells through inhibition of reduced $\mathrm{GSH}$ and induction of mitochondria-involved apoptosis 
monitored by molecular biochemistry technics. ${ }^{24}$ In this study, we investigated the toxicity of AgNP in human Chang liver cell, with NMR-based metabolomics approaches. To specify metabolic changes caused by AgNP in the Chang liver cell, AgNP was treated to the cell with and without antioxidant $N$-acetylcysteine (NAC) while cell culture period. HR-MAS NMR was utilized to measure the metabolic profiles in the intact cell and the result was analyzed by the target profiling and pattern recognition methods. The results showed that pyruvate, choline, adipate, glutamate, glycerol, leucine, isoleucine, valine, hypoxanthine sn-glycero-3-phosphochline and o-phosphocholine were increased and glutathione, lactate, inosine and taurine were decreased by the AgNPs. The result might suggest that the ROS could reduce the GSH which is internal antioxidant in the liver cell and enhance the conversion of pyruvate from the lactate and the taurine. The result also showed that several amino acids are related to the depletion of the GSH.

\section{Experimental}

Cell Culture and Sample Collection. Human Chang liver cells were obtained from the American Type Culture Collection (RocKville, MD, USA) and maintained at $37{ }^{\circ} \mathrm{C}$ in an incubator with a humidified atmosphere of $5 \% \mathrm{CO}_{2}$ and cultured in RPMI-1640 medium containing 10\% heat inactivated fetal calf serum, streptomycin $(100 \mu \mathrm{g} / \mathrm{mL})$ and penicillin (100 units/mL). Cells were treated with AgNPs and $\mathrm{AgNO}_{3}$ at various concentrations. ${ }^{24}$

Sample Preparation and NMR Experiments. All cell samples were stored in $-80{ }^{\circ} \mathrm{C}$ freezer until analysis. Cells were divided into three groups, which were control, AgNP treated and AgNP with NAC treated and each group include four samples. Each cell sample was weighted $25 \mathrm{mg}$ and loaded into a $4 \mathrm{~mm}$ nano zirconium rotor. Total volume was adjusted to $45 \mu \mathrm{L}$ with deuterium oxide with $20 \mu \mathrm{L}$ deuterium oxide mixed with $5 \mu \mathrm{L} 20 \mathrm{mM} 3$-(Trimethylsilyl) propionic-2,2,3,3- $d_{4}$ acid (TSP), and the final concentration TSP was $4 \mathrm{mM}$. This solvent was transferred into $4 \mathrm{~mm}$ zirconium rotor. A lid was capped as a closure of the rotor and marked at the rotor for the monitoring of spinning speed.

${ }^{1} \mathrm{H}$ NMR measurement was carried out on a $400 \mathrm{MHz}$ Varian Unity-Inova (Varian Inc., Palo Alto, CA) operating at a proton frequency of $400.266 \mathrm{MHz}$. All instruments were equipped with a gHX nanoprobe with a spin rate of $2000 \mathrm{~Hz}$ and regulated temperature at $299 \mathrm{~K}$. Deuterium oxide and TSP- $\mathrm{d}_{4}$ were used as the field-lock frequency and internal chemical shift reference, respectively. Measurement of 1D proton NMR spectra acquired with presaturation and CPMG (Carr-Purcell-Meiboom-Gill) pulse sequence to suppress water signal and presaturation was better in these samples. Presaturated proton 1D NMR spectra were collected spectra width of $6404 \mathrm{~Hz}$, resulting in an acquisition time of $2 \mathrm{~s}$, relaxation time of $1 \mathrm{~s}$, and number of scan was 512 . All data

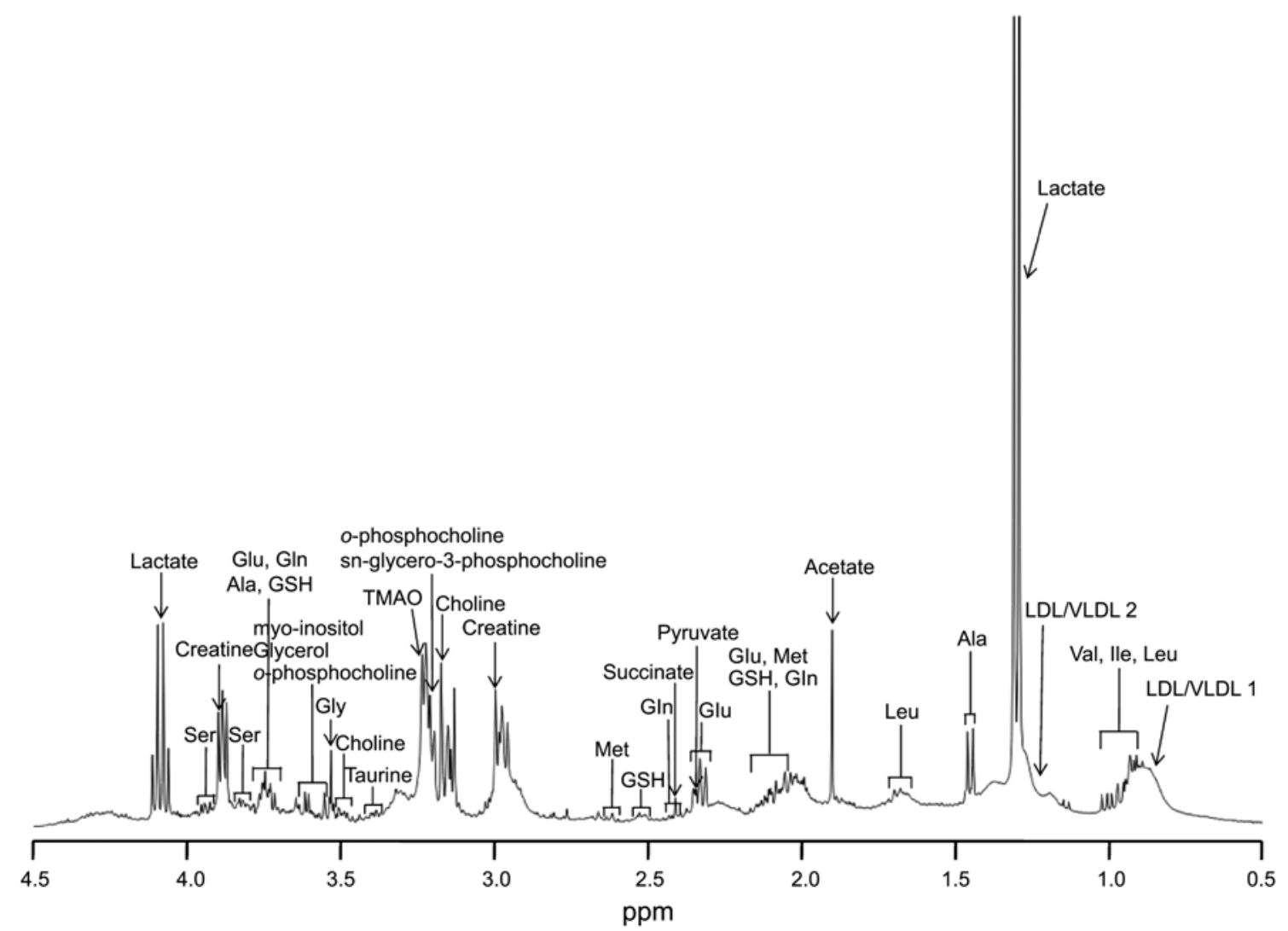

Figure 1. Control sample was selected as an example for detailed signal assignment with TSP $(\delta 0.00 \mathrm{ppm})$. The following metabolites were identified with Chenomx $400 \mathrm{MHz}$ library. Abbreviation: LDL/VLDL, Low-density lipoprotein/Very low-density lipoprotein; Ala, Alanine; Leu, Leucine; Val, Valine; Ile, Isoleucine; Glu, Glutamic acid; Gln, Glutamine; GSH, Glutathione; Met, Methionine; Gly, Glycine; Ser, Serine; TMAO, Trimethylamine N-oxide. 
was referenced to TSP- $d_{4}$ at $\delta 0.00 \mathrm{ppm}$ and was apodized with an exponential function using a line broadening of 0.2 $\mathrm{Hz}$.

NMR Data Processing and Statistical Analysis. All NMR data were Fourier transformed, phased, and baseline was manually corrected using VnmrJ (Varian Inc.). 29 major metabolites were assigned for target profiling using the Chenomx NMR Suite 6.01 software (Chenomx Inc., Canada) and spectra were divided into spectral bins $(0.01 \mathrm{ppm})$ for pattern recognition profiling. Each NMR spectrum was reduced to $0.01 \mathrm{ppm}$ spectral buckets and was normalized to the region of $\delta 0.5-10 \mathrm{ppm}$. The region of residual water $\delta$ 4.6-5 ppm was removed from the each spectrum. Normalized spectra with TSP- $d_{4}$ concentration were superimposed and stacked to compare GSH concentration. The concentration of metabolites was estimated from integration of its peak area and compared with TSP- $d_{4}$ peak using Chenomx NMR suite 6.01 .

Multivariative statistical analyses were established with all samples using SIMCA-P+ 12.0.1 software package (Umetrics, $\mathrm{AB}$, Umeå, Sweden). In this study, orthogonal-projection to latent structure-discriminant analysis (OPLS-DA) was performed on Ctr-scaled spectral data which was carried out in order to improve classification of the different groups of individuals as well as to optimize the identification of changes in cellular metabolites that were unique to a particular group.

\section{Results and Discussion}

NMR Measurement and Assignment. ${ }^{1} \mathrm{H}$ NMR spectra were obtained using presaturation pulse sequence, which provide suppressed water signal. Each spectrum was fourier transformed and manually phased and baseline was corrected. The spectrum was referenced and normalized with the $\mathrm{TSP}-d_{4}$ at $\delta 0.00 \mathrm{ppm}$. Metabolites were identified using the Chenomx $400 \mathrm{MHz}$ library and literature compilations. Figure 1 shows HR-MAS ${ }^{1} \mathrm{H}$ NMR spectra of control Chang liver cell spectra, which represent the general metabolites of cell. Several metabolite classes observed, including amino acids, phospholipids, form of methylated amine moiety such as, trimethylamine- $N$-oxide. The major indentified compounds were lactate, leucine, isoleucine, valine, alanine, acetate, GSH (glutathione), pyruvate, creatine, TMAO (trimethylamine $\mathrm{N}$-oxide), choline, sn-glycerol-3-phosphocholine, glycine, serine, myo-inositol. Among these metabolites, concentrations of chosen major compounds are depicted in Table 1. The concentrations of pyruvate, uracil, valine, isoleucine, leucine, choline, sn-glycero-3-phosphocholine, o-phosphocholine and hypoxanthine were increased by the treatment of the AgNP. One of the greatest increase metabolite is pyruvate, which is the main component for a reaction of Krebs cycle. The convert of pyruvates occure in the matrix of the mitochondrion, which were, provide energy to cell. Similary, change of metabolite in AgNP with NAC group is likewise control groups. Most of metabolites which are included GSH and pyruvate were recovered to the normal cell concentrations. The effect of AgNP induced the ROS was
Table 1. Relative concentration of major metabolites represented in table. The concentrations of metabolites were calculated with integration of peak areas using Chenomx. The values are expressed as mean \pm standard error

\begin{tabular}{|c|c|c|c|}
\hline Metabolite & Control & AgNP & $\begin{array}{l}\text { AgNP with } \\
\text { NAC }\end{array}$ \\
\hline Acetate & $3.618 \pm 0.314$ & $4.479 \pm 0.978$ & $6.142 \pm 0.549$ \\
\hline Acetone & $0.410 \pm 0.055$ & $1.482 \pm 0.415$ & $0.801 \pm 0.176$ \\
\hline Adipate & $1.059 \pm 0.034$ & $1.395 \pm 0.214$ & $1.253 \pm 0.189$ \\
\hline Alanine & $3.952 \pm 0.218$ & $4.849 \pm 0.177$ & $3.692 \pm 0.240$ \\
\hline Choline & $1.409 \pm 0.093$ & $3.065 \pm 0.212$ & $1.759 \pm 0.109$ \\
\hline Creatine & $3.090 \pm 0.175$ & $3.988 \pm 0.247$ & $3.675 \pm 0.268$ \\
\hline Glutamate & $6.983 \pm 0.623$ & $7.806 \pm 0.540$ & $7.352 \pm 0.424$ \\
\hline Glutamine & $1.752 \pm 0.081$ & $1.840 \pm 0.136$ & $2.069 \pm 0.198$ \\
\hline Glutathione & $1.435 \pm 0.148$ & $1.248 \pm 0.087$ & $1.824 \pm 0.163$ \\
\hline Glycerol & $1.371 \pm 0.071$ & $1.783 \pm 0.190$ & $1.882 \pm 0.214$ \\
\hline Glycine & $1.934 \pm 0.144$ & $1.572 \pm 0.079$ & $2.032 \pm 0.388$ \\
\hline Hypoxanthine & $1.759 \pm 0.155$ & $2.916 \pm 0.154$ & $1.655 \pm 0.426$ \\
\hline Inosine & $0.602 \pm 0.128$ & $0.332 \pm 0.072$ & $0.263 \pm 0.075$ \\
\hline Isoleucine & $1.473 \pm 0.054$ & $2.359 \pm 0.100$ & $1.795 \pm 0.205$ \\
\hline Lactate & $51.218 \pm 1.552$ & $31.917 \pm 2.435$ & $41.727 \pm 4.473$ \\
\hline Leucine & $3.315 \pm 0.078$ & $5.005 \pm 0.273$ & $4.246 \pm 0.512$ \\
\hline Methionine & $0.762 \pm 0.027$ & $0.865 \pm 0.086$ & $0.821 \pm 0.092$ \\
\hline$o$-Phosphocholine & $1.045 \pm 0.236$ & $2.275 \pm 0.200$ & $1.804 \pm 0.373$ \\
\hline Phenylalanine & $0.451 \pm 0.020$ & $0.464 \pm 0.105$ & $0.484 \pm 0.041$ \\
\hline Pyruvate & $0.646 \pm 0.105$ & $3.446 \pm 0.639$ & $0.785 \pm 0.120$ \\
\hline Serine & $3.021 \pm 0.188$ & $3.488 \pm 0.460$ & $4.081 \pm 0.830$ \\
\hline Succinate & $0.252 \pm 0.026$ & $0.244 \pm 0.007$ & $0.281 \pm 0.012$ \\
\hline Taurine & $1.421 \pm 0.237$ & $0.793 \pm 0.037$ & $1.342 \pm 0.239$ \\
\hline $\begin{array}{l}\text { Trimethylamine } \\
\mathrm{N} \text {-oxide }\end{array}$ & $1.119 \pm 0.058$ & $0.965 \pm 0.096$ & $1.026 \pm 0.081$ \\
\hline Tyrosine & $0.599 \pm 0.048$ & $0.634 \pm 0.075$ & $0.666 \pm 0.052$ \\
\hline Uracil & $1.486 \pm 0.149$ & $4.211 \pm 0.443$ & $1.989 \pm 0.089$ \\
\hline Valine & $1.514 \pm 0.040$ & $2.284 \pm 0.125$ & $1.786 \pm 0.173$ \\
\hline myo-Inositol & $1.260 \pm 0.220$ & $1.675 \pm 0.105$ & $1.366 \pm 0.184$ \\
\hline $\begin{array}{l}\text { sn-Glycero-3- } \\
\text { phosphocholine }\end{array}$ & $1.043 \pm 0.086$ & $2.620 \pm 0.280$ & $1.402 \pm 0.276$ \\
\hline
\end{tabular}

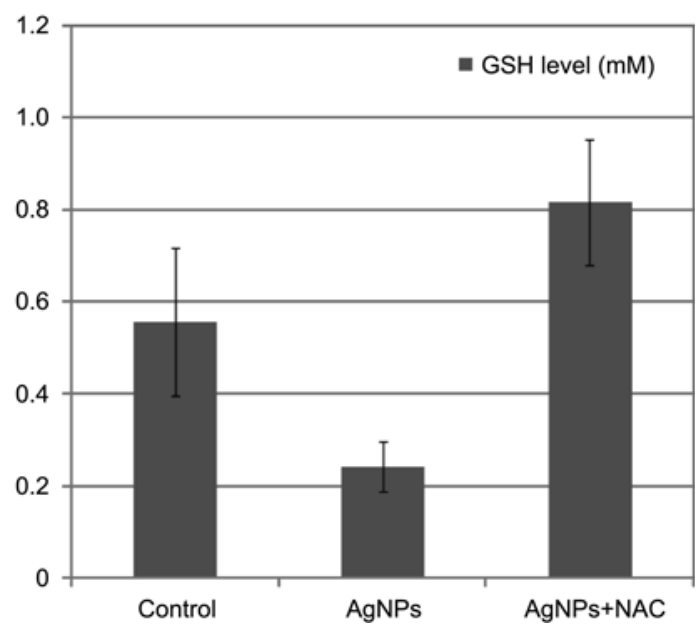

Figure 2. The levels of GSH in control, AgNP and AgNP with NAC group. The data is mean values with standard error. Top: AgNP with NAC group, middle: AgNP group, bottom: control. 


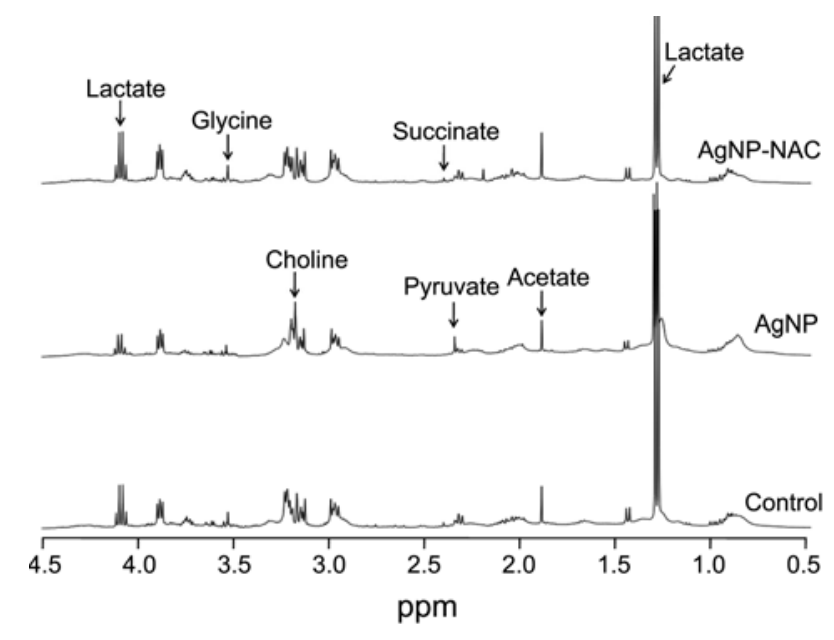

Figure 3. Representative ${ }^{1} \mathrm{H}$ NMR spectra of control, AgNP and AgNP with NAC group. The metabolites which showed major differences were marked on spectrum.

inhibited by NAC, a synthetic antioxidant. The acetate is unlike other metabolites, which of concentration is much higher than those of other groups. In contrast, the decrease of metabolites are glycine, GSH, taurine, TMAO, tyrosine and lactate. Specially, GSH is dramatically decreased in AgNP group compared to control group. In addition, AgNP with NAC group closely similar with the control group. Figure 2 describe the mean GSH level with standard error bar. The amount of the GSH level was decreased less than half by the treatment of AgNPs but the level was recovered by the pretreatment of NAC. Figure 3 represent part of the NMR spectrum including major signals which were changed by the treatment of AgNPs.

Multivariate Analysis. To multivariative analysis, ${ }^{1} \mathrm{H}$ spectra were binned $0.01 \mathrm{ppm}$ wide fragments between $\delta 0.5$ $10 \mathrm{ppm}$, which is, excluded water region $\delta 4.6-5 \mathrm{ppm}$. PCA was carried out on $\mathrm{Ctr}$-scaled data to distinguish any difference between the three groups. OPLS-DA applied for the separation three groups which in hotelling's $\mathrm{T}$ region with $95 \%$ confidence interval for the modeled variation. Figure 4 shows the OPLS-DA result as score plot, for the first two principal components $(\mathrm{t} 1, \mathrm{t} 2)$ from the presaturation

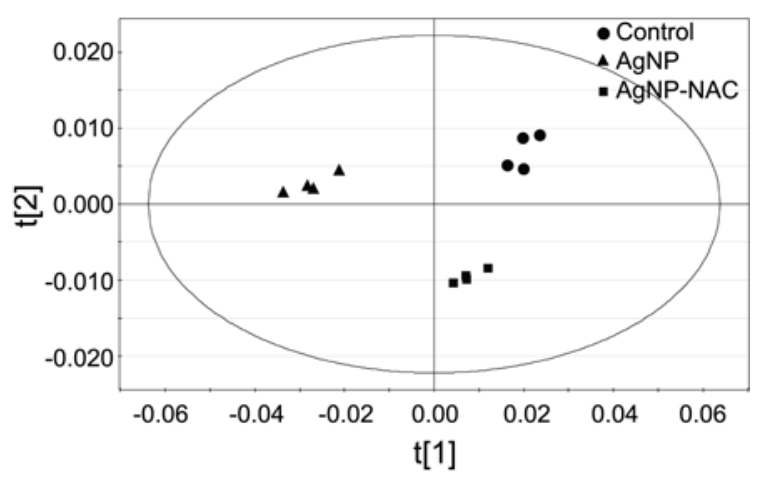

Figure 4. OPLS-DA score plot (comp. 1 vs. comp. 2) obtained from the NMR spectra of 12 cell samples using SIMCA-P+12.0. 12 samples were classified into three groups. $\bullet$ : control; $\mathbf{\Delta}$ : AgNP group; $\mathbf{\square}$ : AgNP with NAC group.
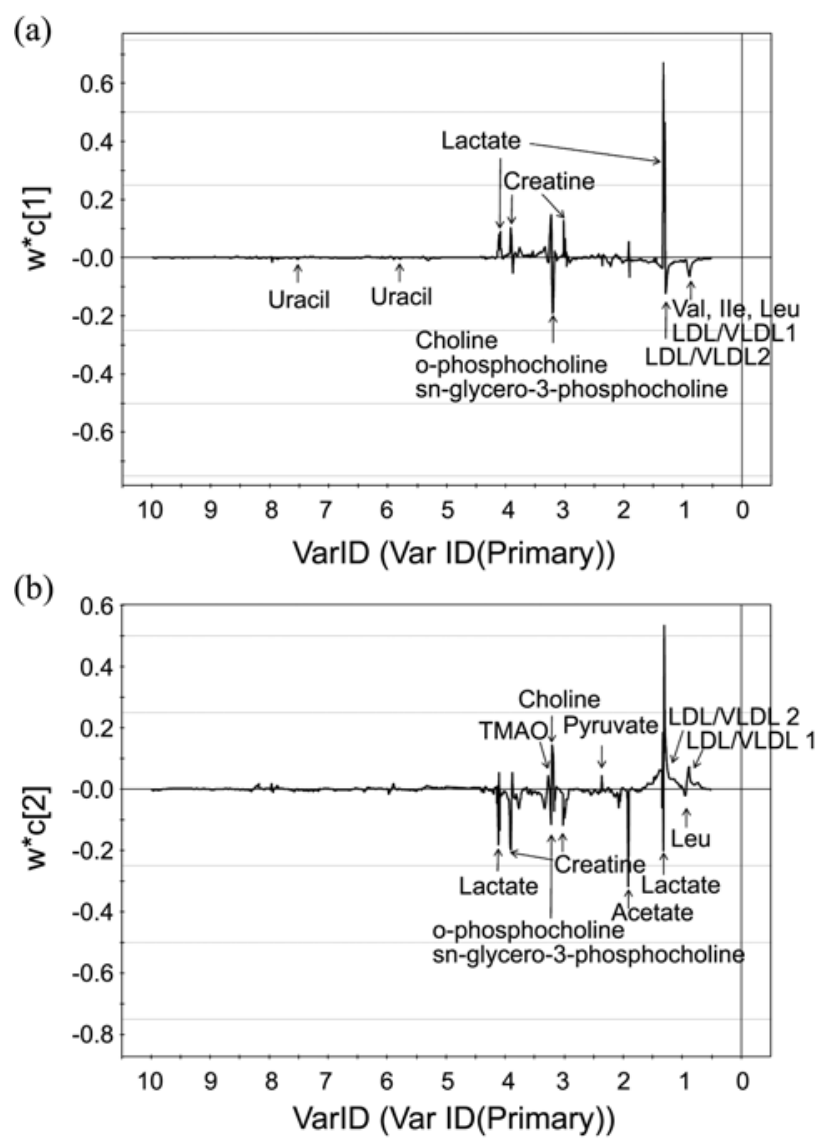

Figure 5. OPLS-DA loading plots based on $1 \mathrm{H}$ NMR spectra of component 1 and 2. (a) OPLS-DA loading plot of component 1, (b) OPLS-DA loading plot of component 2 . There were several major components that discriminated between control and AgNP group; lactate, pyruvate, choline, acetate, and GSH.

spectra of the three groups. The three groups are named control (•), AgNPs ( $\bullet$ ) and AgNPs with NAC ( $\boldsymbol{\bullet})$ groups. The values of $\mathrm{R}^{2}$ were 0.991 and 0.967 , respectively, and $\mathrm{Q}^{2}$ describing the predictability of the model was 0.541 . The corresponding loading plot shows same tendency in their changes of metabolite with Table 1 on the responsible for the grouping. To identify the major contribution to the separation, we generated the OPLS-DA loading plot. Figure 5 presents the loading plots from the analysis of ${ }^{1} \mathrm{H}$ NMR spectra of cell samples for control and AgNP groups. The remarkable differences between the control and AgNP group were achieved along the first principal components. The loading plot shows that the greatest change in response to AgNP induced includes the elevation of lipids, adipate, as well as the decrease of lactate, acetate, creatine, choline, GSH and the results consist with the analysis in Table 1.

\section{Conclusion}

We reported the evidence for a molecular mechanism via which AgNPs induce cell damage via the generation of ROS and the induction of apoptosis via mitochondria-dependent and caspase-dependent pathways mediated by JNK. 


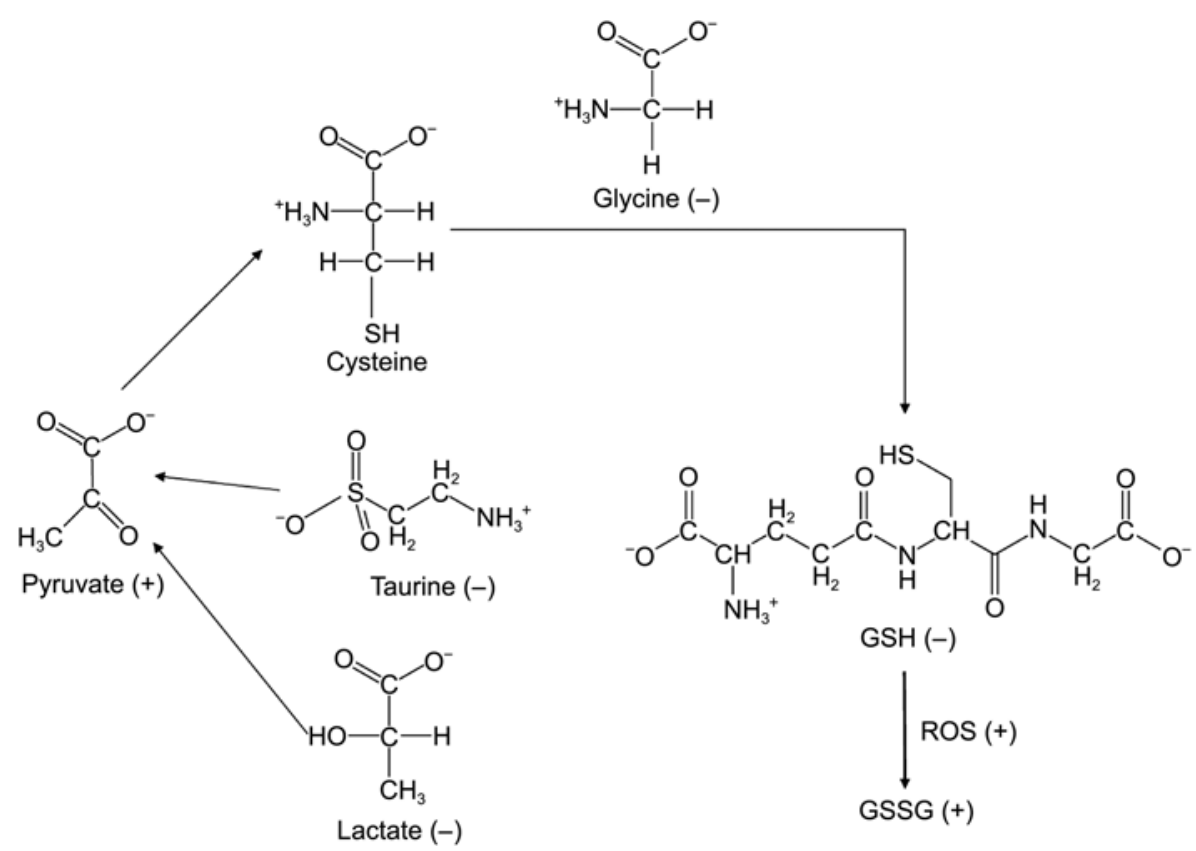

Figure 6. Summary of metabolites that affected by the AgNPs. The (+) indicates metabolite which was increased and (-) indicates that the metabolite shows reduction.

Additionally, ROS generation in AgNPs-treated cells was decreased by pre-treatment of cells with NAC in our previous report. ${ }^{24}$ It was discussed as GSH acts directly as a scavenger of ROS and as a substrate for GSH peroxidase to reduce hydrogen peroxide. ${ }^{25-27}$

However, we could not identify the effects to other metabolites by the AgNPs in our previous work. In this work, we identified many metabolites affected in their concentration through the treatment of AgNPs. Moreover, we conformed that the most metabolites were recovered by the pre-treatment of NAC, external antioxidant reagent. Most dramatically changed metabolites were pyruvate and lactate, which is a key joining in several metabolic pathways. The concentration of lactate was decreased and pyruvate was increased by the treatment of AgNPs. The result implies that the lactate converted to the pyruvate and ROS might play a role as a trigger for this reaction because the levels of both metabolites were recovered with pre-treatment of the NAC. The result also suggests that the needs of energy in the liver cell could prefer generation of pyruvate than lactate when treated with AgNPs.

The AgNPs increased the levels of choline, o-phosphocholine, and sn-glycerol-3-phosphocholine, which are involved in phospholipid pathways. The Phospholipids membranes might be attacked by the ROS and the levels of those metabolites could be affected. Several amino acids were changed in their concentration by the treatment of AgNPs. In the amino acids, glutamate is a key junction in several metabolic pathways, the concentration of it was decreased, and we detected a minor decrease in the level of glutamine, which is closely related to glutamate in the several pathways. Moreover, the levels of valine, isoleucine, leucine, and alanine were enhanced. The concentration of these amino acids might be related to level of the pyruvate and they show same tendency with pyruvate. On the contrary, glycine shows low level of its concentration with the treatment of AgNPs, which implies the conversion to the GSH. Taurine also shows a low level in the high ROS state and it might be converted to the pyruvate or some other metabolites.

Figure 6 shows the summary of metabolites that affected by the AgNPs in our investigation. The consumption of GSH by the ROS enhances the conversion of lactate and taurine to pyruvate and the synthesis of the GSH with the reduction of glycine.

Acknowledgments. This work was supported for two years by Pusan National University Research Grant.

\section{References}

1. Medina, C.; Santos-Martinez, M. J.; Radomski, A.; Corrigan, O. I.; Radomski, M. W. British Journal of Pharmacology 2007, 150, 552-558.

2. Oberdörster, G.; Oberdörster, E.; Oberdörster, J. Environmental Health Perspectives 2005, 113, 823-839.

3. Lewinski, N.; Colvin, V.; Drezek, R. Small 2008, 4, 26-49.

4. Sur, I.; Cam, D.; Kahraman, M.; Baysal, A.; Culha, M. Nanotechnology 2010, 21, 175104-175113.

5. Nel, A.; Xia, T.; Mädler, L.; Li, N. Science 2006, 311, 622-627.

6. Ahamed, M.; AlSalhi, M. S.; Siddiqui, M. K. J. Clinica Chimica Acta 2010, 411, 1841-1848.

7. AshaRani, P. V.; Mun, G. L. K.; Hande, M. P.; Valiyaveettil, S. ACS Nano 2009, 3, 279-290.

8. Cohen, M. S.; Stern, J. M.; Vanni, A. J.; Kelley, R. S.; Baumgart, E.; Field, D.; Libertino, J. A.; Summerhayes, I. C. Surgical Infections 2007, 8, 397-403.

9. Fu, J.; Ji, J.; Fan, D.; Shen, J. Journal of Biomedical Materials Research - Part A 2006, 79, 665-674. 
10. Xu, X.; Yang, Q.; Bai, J.; Lu, T.; Li, Y.; Jing, X. Journal of Nanoscience and Nanotechnology 2008, 8, 5066-5070.

11. Chaby, G.; Viseux, V.; Poulain, J. F.; De Cagny, B.; Denoeux, J. P.; Lok, C. Annales de dermatologie et de vénéréologie 2005, 132, 891-893.

12. Trop, M.; Novak, M.; Rodl, S.; Hellbom, B.; Kroell, W.; Goessler, W. The Journal of Trauma 2006, 60, 648-652.

13. Kim, S.; Choi, J. E.; Choi, J.; Chung, K. H.; Park, K.; Yi, J.; Ryu, D. Y. Toxicolgy In Vitro 2009, 23, 1076-1084.

14. Martindale, J. L.; Holbrook, N. J. Journal of Cellular Physiology 2002, 192, 1-15.

15. Thannickal, V. J.; Fanburg, B. L. American Journal of Physiology - Lung Cellular and Molecular Physiology 2000, 279, L1005L1028.

16. Sies, H. Free Radical Biology \& Medicine 1999, 27, 916-921.

17. Nicholson, J. K.; Connelly, J.; Lindon, J. C.; Holmes, E. Nature Reviews Drug Discovery 2002, 1, 153-161.

18. Nicholson, J. K.; Lindon, J. C. Nature 2008, 455, 1054-1056.

19. Kwon, B.; Kim, S.; Kim, S.; Lee, D. K.; Park, Y. J.; Kim, M. D.; Lee, J. S.; Kim, S. Forensic Science International 2011, accepted.
20. Holmes, E.; Nicholls, A. W.; Lindon, J. C.; Ramos, S.; Spraul, M.; Neidig, P.; Connor, S. C.; Connelly, J.; Damment, S. J. P.; Haselden, J.; Nicholson, J. K. NMR in Biomedicine 1998, 11, 235244.

21. Bollarda, M. E.; Murrayb, A. J.; Clarkeb, K.; Nicholson, J. K.; Griffin, J. L. FEBS Letters 2003, 553, 73-78.

22. Waters, N. J.; Holmes, E.; Willians, A.; Waterfield, C. J.; Farrant, R. D.; Nicholson, J. K. Chemical Research in Toxicology 2001, 14, 1401-1412.

23. Lehnhardt, F. G.; Bock, C.; Röhn, G.; Ernestus, R. I.; Hoehn, M. NMR in Biomedicine 2005, 18, 371-382.

24. Piao, M. J.; Kang, K.A.; Lee, I. K.; Kim, H. S.; Kim, S.; Choi, J. Y.; Choi, J.; Hyun, J. W. Toxicology Letters 2011, 201, 92-100.

25. Habib, G. M.; Shi, Z. Z.; Lieberman, M. W. Free Radical Biology \& Medicine 2007, 42, 191-201.

26. Peña-Llopis, S.; Ferrando, M. D.; Peña, J. B. Aquatic Toxicology 2003, 65, 337-360.

27. Vairetti, M.; Griffini, P.; Pietrocola, G.; Richelmi, P.; Freitas, I. Free Radical Biology \& Medicine 2001, 31, 954-961. 